\title{
Changes in trunk orientation do not induce asymmetries in covert orienting
}

\author{
David A. Westwood • Stephanie A. H. Jones • \\ Christopher D. Cowper-Smith • Raymond M. Klein
}

Published online: 11 May 2013

(C) Psychonomic Society, Inc. 2013

\begin{abstract}
We explored the effect of trunk orientation on responses to visual targets in five experiments, following work suggesting a disengage deficit in covert orienting related to changes in the trunk orientation of healthy participants. In two experiments, participants responded to the color of a target appearing in the left or right visual field following a peripheral visual cue that was informative about target location. In three additional experiments, participants responded to the location (left/right) of a target using a spatially compatible motor response. In none of the experiments did trunk orientation interact with spatial-cuing effects, suggesting that orienting behavior is not affected by the rotation of the body relative to the head. Theoretical implications are discussed.
\end{abstract}

\section{Keywords Attention $\cdot$ Space $\cdot$ Posture $\cdot$ Trunk $\cdot$ Vision}

We need to be able to accurately locate objects relative to ourselves and other objects in the environment for many of our daily activities, such as in navigating around furniture, reaching to grasp a coffee cup, or identifying the source of an unfamiliar sound. Thus, it is not surprising that neurons in many regions of the human cortex are sensitive to the spatial locations of sensory events. Neurophysiological research in macaques (Avillac, Denève, Olivier, Pouget, \& Duhamel, 2005; Batista et al., 2007; Buneo, Jarvis, Batista, \& Andersen, 2002; Caminiti, Johnson, Galli, Ferraina, \& Burnod, 1991; Lacquaniti, Guigon, Bianchi, Ferraina, \& Caminiti, 1995) and both neuroimaging (Wu \&

D. A. Westwood $(\bowtie) \cdot$ S. A. H. Jones

School of Health and Human Performance, Dalhousie University, Halifax, NS B3H 3J5, Canada

e-mail: david.westwood@dal.ca

C. D. Cowper-Smith $\cdot$ R. M. Klein

Department of Psychology and Neuroscience, Dalhousie

University, Halifax, NS, Canada
Hatsopoulos, 2006, 2007) and behavioral (Andersen, Snyder, Bradley, \& Xing, 1997; Culham \& Kanwisher, 2001; Graziano \& Gross, 1998; Jones \& Henriques, 2010; Mountcastle, Lynch, Georgopoulos, Sakata, \& Acuna, 1975; Wandell, 1999) studies in humans have shown that the central nervous system (CNS) uses multiple reference frames (Previc, 1990, 1998) to code the locations of sensory events. The present investigation focused on the bodycentered reference frame, which has been revealed through biases of spatial processing in patients suffering from hemispatial neglect (Bartolomeo \& Chokron, 1999, 2002; De Renzi, 1982).

Hemispatial neglect (HSN) is a syndrome characterized by a spatially graded impairment in detecting and responding to sensory information (Butler, Lawrence, Eskes, \& Klein, 2009). Hemispatial neglect typically affects the left side of space following a lesion to the right cortex (although this is not always the case; Bowen, McKenna, \& Tallis, 1999). Some lines of evidence have suggested that the spatial impairment observed in HSN may arise from a rightward shift in the egocentric representation of body position with respect to objects in the environment (e.g., Bisiach, Capitani, \& Porta, 1985; Karnath, 1994; Karnath, Schenkel, \& Fischer, 1991; Rorden, Karnath, \& Driver, 2001). For example, Karnath et al. (1991) found that rotating the trunk to the left eliminated the left-right asymmetry in saccadic onset times (i.e., the longer reaction times [RTs] to stimuli presented in the left visual field when the head and body were oriented forward) for patients with left HSN, an effect that did not occur in stroke patients without neglect or in healthy control participants. Karnath (1994) later reported that left-side caloric stimulation (i.e., the infusion of cool water into one ear) and neck tendon vibration improved a $15^{\circ}$ rightward bias in perceptual "straight ahead" in three patients with left HSN. No effects of caloric stimulation or neck vibration alone were found in healthy control participants, suggesting that the improvements seen in the HSN 
patients were not due to general effects on covert orienting of spatial attention (i.e., the intentional or sensory eventdriven allocation of attentional resources to specific regions of space in the absence of changes in the orientation of the sensory apparatus; Klein, 2004). Instead, the benefits seen in HSN appeared to reflect the amelioration of a pathological asymmetry in a body-centered reference frame that is influenced by sensory input from neck proprioceptors and the vestibular system (Karnath, 1994).

In contrast to Karnath et al. (1991) and Karnath (1994), Grubb and Reed (2002) reported an effect of trunk orientation on covert orienting in healthy controls. Grubb and Reed (2002) argued that previous studies did not isolate the attentional mechanism that is most strongly impaired in HSN: the disengagement of attention from one side of space that is necessary to shift attention toward the location of a new salient event (Posner, Walker, Friederiech, \& Rafal, 1984). An impairment of attentional disengagement, inferred from excessively long response times for invalidly cued targets in the contralesional half of space (Losier \& Klein, 2001), has been proposed to be characteristic of HSN patients (Posner et al., 1984). To address this issue, Grubb and Reed (2002) presented healthy control participants with a cue-target task in which informative peripheral cues were used to generate orienting. In such a task, it is conventional to describe trials as valid when the target appears at the cued location and invalid when the target appears at the opposite, un-cued location. In Fig. 1, we have adapted the results of Grubb and Reed (2002), presenting them according to the graphical convention from Losier and Klein (2001, Figs. 1, 2, 3 and 4) to emphasize how trunk orientation (panels) and the visual field in which the target was presented (line type) interacted with the cue-target relationship. A comparison of the two lines in the first panel (left trunk orientation) shows that the cuing effect (invalid minus valid reaction times (RTs)) was smaller when the target was presented in the left visual field (LVF, dotted line) versus the right visual field (RVF, solid line); in particular, although the LVF and RVF RTs were similar for valid trials, RTs were substantially slower in the RVF than in the LVF for invalid trials (creating a larger cuing effect in the RVF). Because the detection of invalidly cued targets in the RVF was impaired (i.e., relative to LVF) only in the left trunk orientation, Grubb and Reed (2002) concluded that changes in trunk orientation resulted in an asymmetrical disengagement effect in healthy adults, similar to that observed in neglect patients. ${ }^{1}$ However, upon considering all three

\footnotetext{
${ }^{1}$ Grubb and Reed (2002) suggested that a leftward trunk orientation resulted in a problem disengaging from the LVF in order to process RVF targets. They suggested that, even though it is inverted, this pattern of RTs in their healthy participants was similar to the rightward bias experienced by patients with right hemisphere damage, which results in a problem disengaging from the RVF in order to process LVF targets.
}

panels in the figure, we can see that with normal (center panel) and rightward trunk orientations (right panel), participants show a pattern that is opposite from that with left trunk orientation; this pattern may suggest a difficulty disengaging from RVF cues in order to orient to LVF targets (RTs to invalidly cued targets in the LFV were slow relative to those in the RVF). Taking the results at face value (while adhering to the Posnerian disengagement idea), we suggest a description different from that offered by Grubb and Reed (2002): In normal participants facing straight forward, a small disengage deficit related to trunk orientation affects the detection of invalidly cued LVF targets; this is unaffected by a rightward trunk orientation, but is reversed (to become a disengage deficit for the detection of RVF targets) during left trunk orientation. Overall, the changes in trunk orientation did not create neglect-like patterns in Grubb and Reed's (2002) normal participants (as those researchers suggested), but instead produced an effect on spatial attention similar to that found in patients with neglect (i.e., turning left ameliorates neglect).

Because the task used by Grubb and Reed (2002) required participants to indicate the location of a visual target using a response that was spatially aligned with the arrangement of stimuli (i.e., participants pushed the left button on a computer mouse if the target appeared on the left side of space, and the right button for targets on the right side of space), the performance effects generated by the informative peripheral cues could have reflected either of two possible mechanisms (Klein, 2004; see, especially, his note 1): response preparation or covert orienting. Here, we attempted to isolate covert orienting mechanisms (which, if present, might be consistent with an attentional bias such as that observed in HSN), by requiring participants to respond to a nonspatial feature of the target (its color) using spatially orthogonal response keys in two of the five experiments $(1$ and 4$){ }^{2}$

In Experiment 1, the participants completed a cue-target task in which they reported the color of the target object using response keys spatially orthogonal (Spence \& Driver, 1997 ) to the possible target locations (left and right visual field). As in Grubb and Reed (2002), cue location predicted target location most of the time and could be valid, invalid, or neutral, and the task was performed with the trunk aligned with the head or rotated $15^{\circ}$ left or right.

\footnotetext{
${ }^{2}$ It may appear odd to the reader that we did not first attempt to replicate the methodology of Grubb and Reed (2002). However, their use of informative spatial cues and responses that were orthogonal to the cuing dimension (left/right) is justification in itself as to why we did not begin this research by attempting to strictly replicate their methods. Nothing would have been gained or learned from using their methodology, even in the event that we were able to replicate the significant three-way interaction that they reported. Incidentally, Experiment 5 of the present study directly replicated the methodology of Grubb and Reed, yet failed to show the three-way interaction between trunk orientation, cue-target relationship, and visual field on which their arguments were based.
} 
Fig. 1 Figure adapted from Grubb and Reed (2002): Mean reaction times broken down by trunk orientation (left, center, right), cue-target relationship (valid, invalid), and visual field (left, right). The data in the figure are from "Trunk Orientation Induces Neglect-Like Lateral Biases in Covert Attention," by J. D. Grubb and C. L. Reed, 2002, Psychological Science, 13, pp. 553-556. Copyright 2002 by the American Psychological Society. Adapted with permission

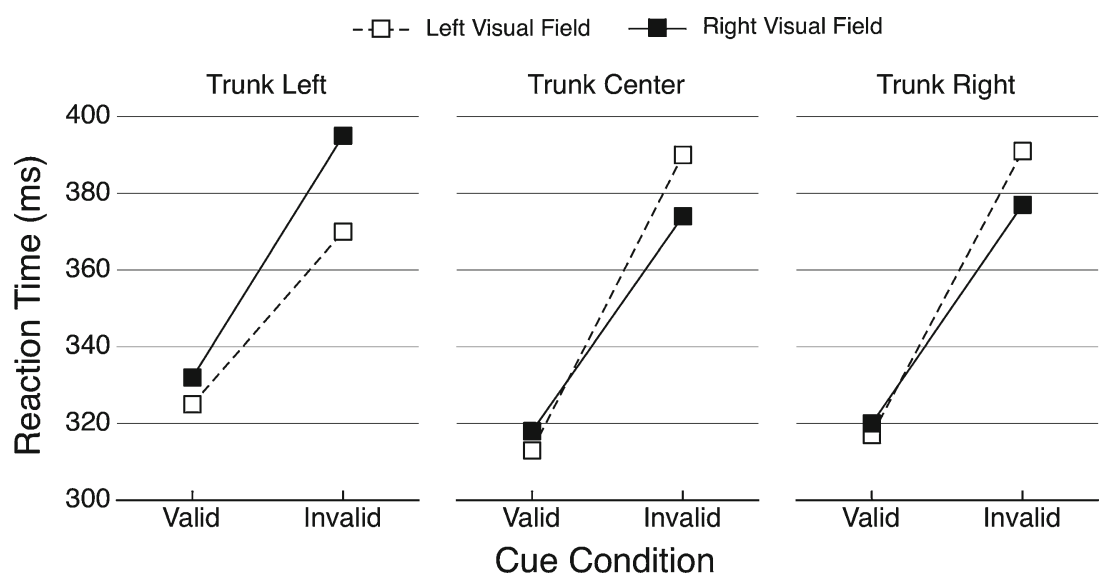

Unlike in the results of Grubb and Reed (2002), trunk orientation did not interact with cuing effects or target location in Experiment 1. In Experiment 2, we adopted the same spatial responses used by Grubb and Reed (2002), such that participants reported the location of the target object by pressing keys that were spatially aligned with the visual targets. Experiment 2 also failed to reveal any interactions between trunk orientation and cuing effects or target location. In Experiment 3, participants once again reported the location of the target object (using the same motor response as in Exp. 2), but two additional trunk rotations were included in

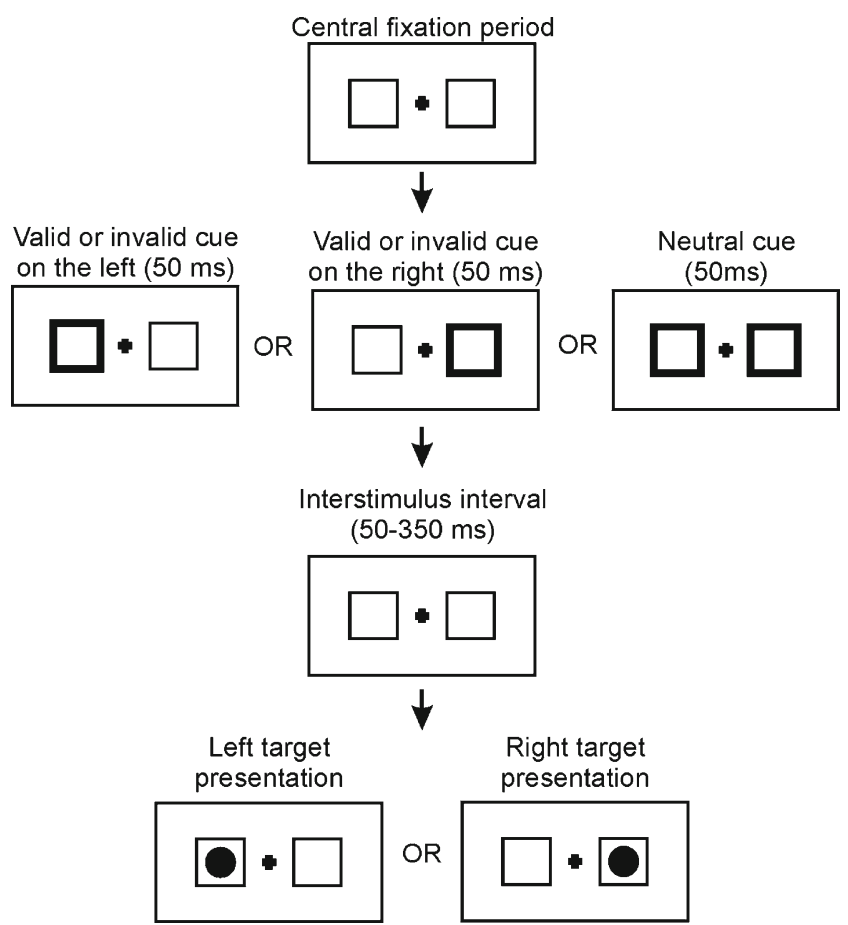

Fig. 2 Schematic of the trial sequence used in all four experiments. In Experiments 1 and 4, participants indicated the color of the presented target (blue or red). In Experiments 2 and 3, participants indicated the location of the presented target (left or right visual field) order to determine whether more extreme trunk rotations might restore the effects reported by Grubb and Reed (2002). Additionally, as in Grubb and Reed's (2002) study, a single cue-target stimulus onset asynchrony (SOA) was used $(200 \mathrm{~ms})$, so as to match more closely the total number of trials used in the original report. Experiment 4 replicated the methods of Experiment 3, except that participants were asked once again to report the color of the target instead of its location. No interactions between trunk orientation and cuing effects were revealed in Experiment 3 or 4. In Experiment 5, we carried out a strict replication of all reported details of Grubb and Reed's (2002) methodology, but found no evidence of the critical three-way interaction between trunk orientation, cue validity, and target location. These findings call into question Grubb and Reed's (2002) conclusion that trunk rotation influences the disengagement of covert spatial attention in an asymmetrical manner; indeed, our results provided no support for any relationship between trunk rotation and the disengagement of spatial attention, as evidenced through the impaired detection of invalidly cued targets.

\section{Experiment 1}

In Experiment 1, we sought to determine the effect of trunk orientation on the disengagement and shifting of spatial attention, independent of possible effects of trunk orientation on lateralized motor preparation. We adapted the task used by Grubb and Reed (2002), such that participants were required to respond to the color of the target (i.e., a nonspatial feature of the target, as opposed to the location of the target) using computer keyboard response keys that were oriented orthogonal to the arrangement of possible visual fields (i.e., front and back, as opposed to left and right). In contrast with Grubb and Reed (2002), who employed a single cue-target asynchrony of $200 \mathrm{~ms}$, three 
Fig. 3 Experiment 1 mean reaction times for each trunk orientation (panels: left, center, right), cue-target relationship (valid, neutral, invalid), and visual field (left, dotted lines and open symbols; right, solid line and symbols)

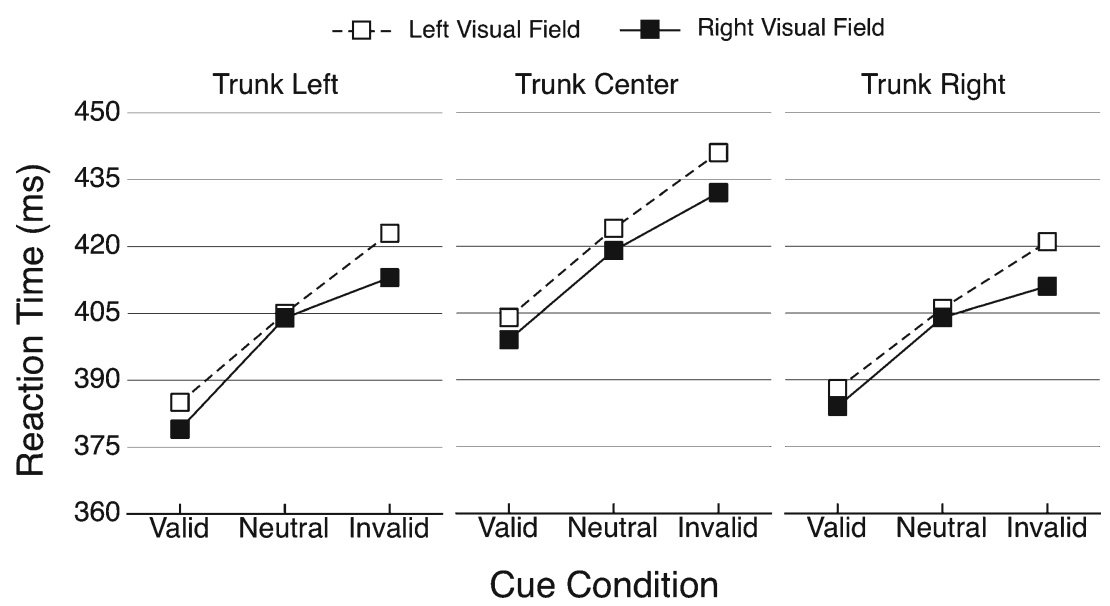

cue-target onset asynchronies were used in Experiment 1 , in order to probe the time course of performance costs and benefits: 100, 200, and $400 \mathrm{~ms}$. Moreover, to control for the alerting properties of lateralized cues, a neutral cue condition consisted of cuing both the left and right locations.

\section{Method}

Participants A total of 24 undergraduate students from the Department of Psychology at Dalhousie University participated in the experiment in exchange for partial course credit; of these, 20 (three male, 17 female) were retained in the analysis. All participants were righthanded (self-report) and had no history of visual, motor or neurological abnormalities. Four participants were excluded from the final analysis; three of them used an incorrect response key for some trials, and one participant responded significantly more slowly than the remainder of participants (the mean RT was more than three standard deviations above the group mean). All participants provided informed consent, and the project was approved by the Dalhousie University Research Ethics board.

Procedure The participants were comfortably seated $57 \mathrm{~cm}$ in front of a 17-in. computer monitor (Samsung SyncMaster $955 \mathrm{MB}$ ) on which all of the stimuli were presented (in Superlab version 2.0, on a Dell Dimension 8300 computer). A chinrest was used to ensure a forward gaze and head direction, despite changes in trunk orientation across blocks of trials. Participants performed a choice RT task (described below) with their trunk in one of three orientations (blocked trials, counterbalanced): $15^{\circ}$ leftward, straight ahead $\left(0^{\circ}\right)$, and $15^{\circ}$ rightward (relative to the computer monitor and head orientation). Trunk orientations were manipulated by changing the direction of the chair according to tape markings measured on the floor and locking the chair in place. The keyboard response keys were mounted directly between the chinrest and the monitor. Participants completed 1,008 trials in total (336 trials for each trunk orientation block).

Choice reaction time task On each trial, participants responded to the color of a target (blue or red) with their
Fig. 4 Experiment 1 mean reaction times for each stimulus onset asynchrony (SOA; panels), cue-target relationship (valid, neutral, invalid), and visual field (left, dotted lines and open symbols; right, solid line and symbols)

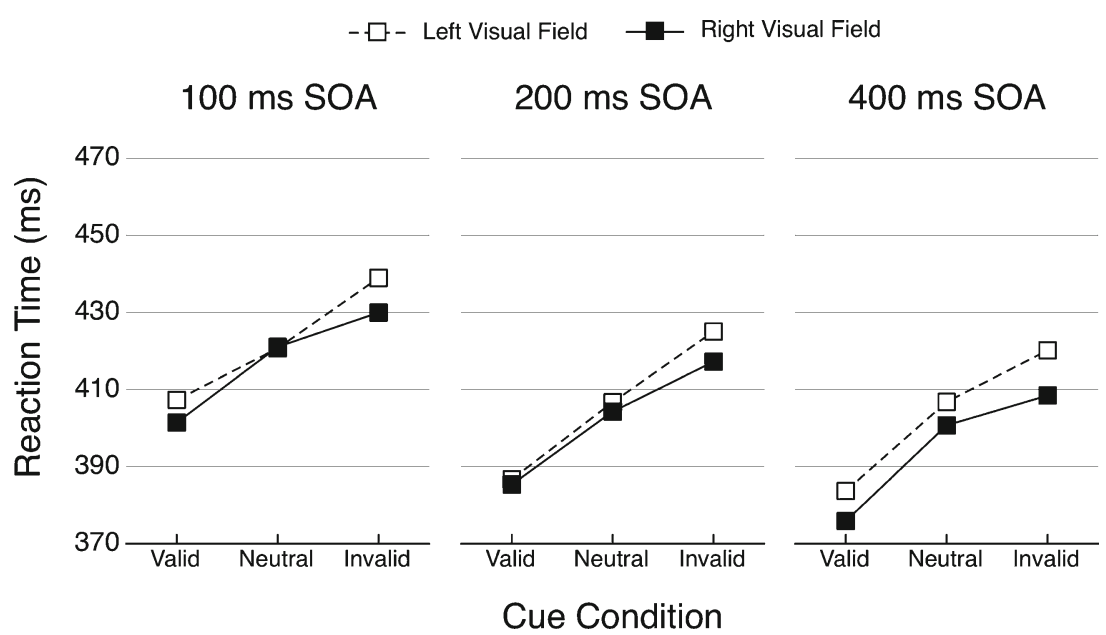


right hand by pressing one of two computer keyboard keys (the "." [period] or "/" [forward slash] keys, respectively). The computer keyboard was turned such that the placement of the keys was orthogonal to the stimulus presentation; the "/" was farther from the body and corresponded to red targets, and the "." was closer to the body, corresponding to blue targets (Grubb \& Reed, 2002, employed the two buttons of a computer mouse held in the right hand). A schematic of the trial sequence is presented in Fig. 2. Three "placeholders" were visible on the computer screen at all times: one fixation cross $\left(0.7 \mathrm{~cm}\right.$ in width; $\left.0.7^{\circ}\right)$ and two target areas in which the targets could appear (peripheral boxes, $1.7 \mathrm{~cm}$ in width; $\left.1.7^{\circ}\right)$. The two peripheral cue boxes were $5 \mathrm{~cm}\left(5^{\circ}\right)$ to the left and right of the central fixation cross. Participants were instructed to maintain fixation on the central fixation cross at all times (monitored by the experimenter). Following a fixation period at the beginning of the trial, participants were cued either to one of the peripheral boxes (the box was "bolded" for $50 \mathrm{~ms} ; 83.3 \%$ of the trials) or to both of them (both boxes were "bolded" for $50 \mathrm{~ms}$, referred to as a "neutral cue"; $16.7 \%$ of all trials). When cued to one of the peripheral boxes, the cue could be "valid," in which case the target appeared in the same location as the previously cued single box (66.7\% of all cues), or "invalid," in which case the target appeared in the location opposite from the previously cued single box (16.7\% of all trials). At this point in the trial, one of three interstimulus intervals (ISIs) was used: 50, 150, or $350 \mathrm{~ms}$ (corresponding to a cue-target SOA of 100, 200 , or $400 \mathrm{~ms})$. These ISIs were used to ensure that participants could not predict when the target would be presented. Following the ISI, a red or blue circle $(50 \%$ probability) appeared inside the left or right target box (50\% probability). Participants were instructed to respond to the color of the target with their right hand as quickly and as accurately as possible, by pressing one of two response keys oriented along the midsagittal axis (i.e., orthogonal to the locations of the targets). Participants completed 50 practice trials to ensure that they understood which keys corresponded to which target colors (trunk orientation was centered for the practice trials). Color names were also printed on the computer board keys as a reminder during the experiment.

Data analysis The dependent measure of interest, reaction time (in ms), was analyzed using a 2 (visual field: left, right) $\times 3$ (trunk orientation: $15^{\circ}$ left, center, $15^{\circ}$ right $) \times$ 3 (cue-target relationship: valid, neutral, invalid) $\times 3$ (SOA: 100, 200, or $400 \mathrm{~ms}$ ) repeated measures analysis of variance (ANOVA; $\alpha=.05$ ). Significant main effects were analyzed post hoc using Bonferroni correction. Greenhouse-Geisser-corrected $p$ values are reported.

\section{Results}

Figure 3 plots the average RTs for each trunk orientation (panels: left, center, or right), cue-target relationship (valid, neutral, or invalid), and visual field (LVF [dotted line and open symbols] or RVF [solid line and symbols]). The average RTs for each SOA, cue-target relationship, and visual field are shown in Fig. 4. Accuracy was high across conditions in Experiment 1 (mean proportion correct $[\mathrm{PC}]=.956$ ).

As expected, we found a significant main effect of cue-target relationship, showing that the informative peripheral cues were successful in capturing attention; increasingly fast RTs were observed for valid $\left(M_{\mathrm{RT}}=\right.$ $390 \mathrm{~ms})$ versus neutral $\left(M_{\mathrm{RT}}=410 \mathrm{~ms}\right)$ versus invalid $\left(M_{\mathrm{RT}}=423 \mathrm{~ms}\right)$ cues $[F(2,38)=165.75, p<.001]$. This pattern of RTs across valid, neutral, and invalid cues was found for all SOA levels $[F(4,76)=2.62, p=.062]{ }^{3}$ suggesting that once captured by a peripheral cue, attention correctly remained at the cued location because it was more likely than the opposite location to contain the target.

Unlike Grubb and Reed (2002), we failed to find a significant three-way interaction between trunk orientation, cue-target relationship and visual field for $\operatorname{RTs}[F(4,76)<1, p=.77]$. Although RTs were longer for the center trunk orientation (center panel, $M_{\mathrm{RT}}=420 \mathrm{~ms}$ ) than for the right trunk orientation (right panel, $M_{\mathrm{RT}}=402 \mathrm{~ms}$ ) only $\left.[F(2,38)=4.77, p=.02)\right]$, this effect did not interact with cue-target relationship $[F(4,76)<1$, $p=.64]$, visual field $[F(2,38)<1, p=.89]$, or $\operatorname{SOA}[F(4,76)<1$, $p=.46$; the non-significant interaction between trunk orientation and SOA is not shown in figures].

As can be seen in Fig. 4, a significant two way interaction between visual field and SOA revealed that participants showed faster RTs for RVF targets (solid lines and symbols, $M_{\mathrm{RT}}=405 \mathrm{~ms}$ ) than for LVF targets (dotted lines and open symbols, $M_{\mathrm{RT}}=411 \mathrm{~ms}$ ), particularly for the longest SOA [100-ms SOA $=5 \mathrm{~ms}$ difference, 200-ms SOA = $4 \mathrm{~ms}$ difference, $400-\mathrm{ms} \mathrm{SOA}=9 \mathrm{~ms}$ difference; $F(2,38)=4.76, p=.04]$. This interaction was not affected by cue-target relationship $[F(2,38)=2.98, p=.07]$ or trunk orientation $[F(2,38)<1$, $p=.89$, the non-significant interaction between trunk orientation and SOA is not shown in figures].

\section{Discussion}

To preclude the possibility that the pattern of results reported by Grubb and Reed (2002) were due to an interaction between trunk orientation and response preparation, in Experiment 1, we rendered the participant's task (color

\footnotetext{
${ }^{3}$ Given the near significance of the omnibus $F$ for this effect, post hoc pairwise comparisons were made. These confirmed that any significant differences that might have arose would have been due to changes in the magnitude of cuing effects across SOAs, rather than to differences in the overall pattern of differences across cue-target relationships.
} 
discrimination) spatially orthogonal to the cuing dimension (left/right). Unlike as reported by Grubb and Reed's (2002) results, Experiment 1 revealed no evidence of impairment in the disengagement of spatial attention as a function of either trunk orientation or visual field; no significant interaction was found between trunk orientation, cue-target relationship, and visual field. However, prior to concluding that the pattern of results reported by Grubb and Reed (2002) was due to an interaction between trunk orientation and response preparation, it was necessary to reinstate their method (i.e., responses that were spatially aligned with target location) to show that their reported effect would emerge with this method. This was the purpose of Experiment 2.

\section{Experiment 2}

In Experiment 2, we more closely replicated the methods of Grubb and Reed's (2002) study, in the expectation that by doing so we would be able to obtain the data pattern that they reported. Consequently, we used the task parameters from Experiment 1, with the exception that participants were asked to respond to the location of targets using congruent, spatially aligned motor responses (i.e., Exp. 2 confounded visual field and the direction of the motor response, as in the Grubb and Reed (2002) experiment).

\section{Method}

Participants A group of 21 naïve undergraduate students (10 male, 11 female) from the Department of Psychology at Dalhousie University participated in the experiment in exchange for partial course credit. All of the participants were right-handed (self-reported) with no history of visual, motor, or neurological abnormalities. All participants provided informed consent, and the project was approved by the Dalhousie University Research Ethics Board.

Procedure, task, and data analysis The remaining methods were identical to those used in Experiment 1, with the following exceptions. First, participants were required to respond to the location of the target, as opposed to its color. Second, the computer keyboard response keys were congruent to the orientation of the visual fields; participants were instructed to press the left key (“.") for targets presented in the LVF and the right key ("/") for targets presented in the RVF.

Results

Figure 5 plots the average RTs for each trunk orientation (panels: left, center, right), cue-target relationship (valid, neutral, invalid), and visual field (LVF, dotted line and open symbols; RVF, solid line and symbols). The average RTs for each SOA, cue-target relationship, and visual field are shown in Fig. 6. Accuracy was high across conditions in Experiment 2 ( $M_{\mathrm{PC}}:$.952).

As expected, RTs were increasingly fast for valid $\left(M_{\mathrm{RT}}=298\right)$ versus neutral $\left(M_{\mathrm{RT}}=319\right)$ versus invalid $\left(M_{\mathrm{RT}}=344\right)$ cues, although this effect was larger in Experiment $2\left(M_{\text {Effect }}=45 \mathrm{~ms}\right)$ than had been observed in Experiment $1\left(M_{\mathrm{Effect}}=33 \mathrm{~ms}\right)$. Unlike in Experiment 1 , a three-way interaction between SOA, cue-target relationship, and visual field on RTs emerged $[F(4,80)=33.36, p=.001]$. Specifically, for valid and neutral trials, RTs to targets presented in the LVF were fastest for the 200-ms SOAs, whereas RTs for targets presented in the RVF were slowest at this SOA. The opposite pattern was obtained on invalid trials.

Critically, however, in Experiment 2 we failed to find a significant three-way interaction between trunk orientation,
Fig. 5 Experiment 2 mean reaction times for each trunk orientation (panels: left, center, right), cue-target relationship (valid, neutral, invalid), and visual field (left, dotted lines and open symbols; right, solid line and symbols)

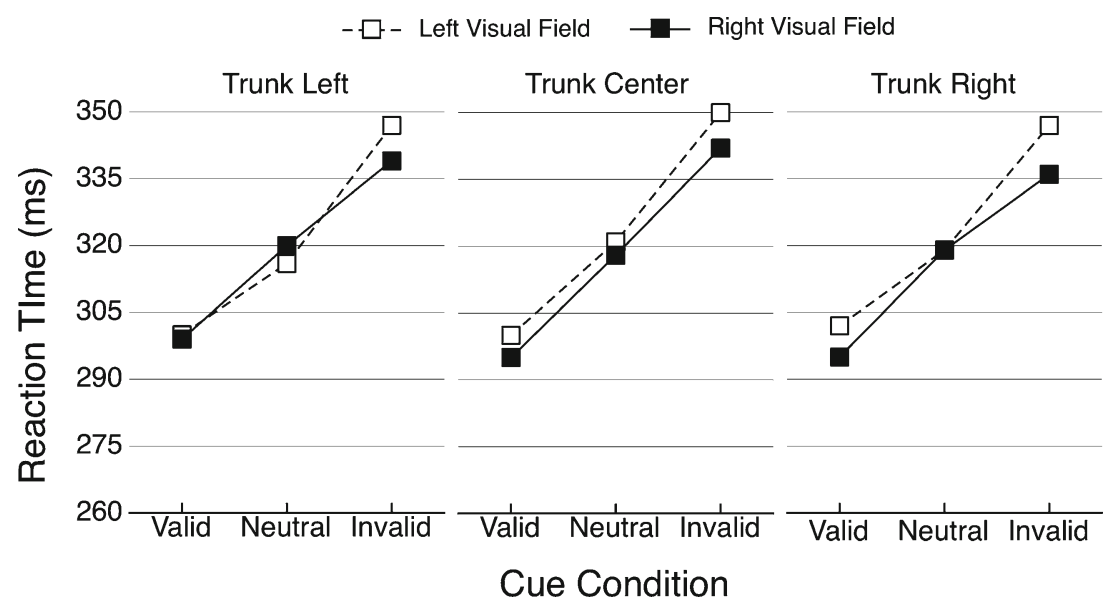


Fig. 6 Experiment 2 mean reaction times for each stimulus onset asynchrony (SOA; panels), cue-target relationship (valid, neutral, invalid), and visual field (left, dotted lines and open symbols; right, solid line and symbols)

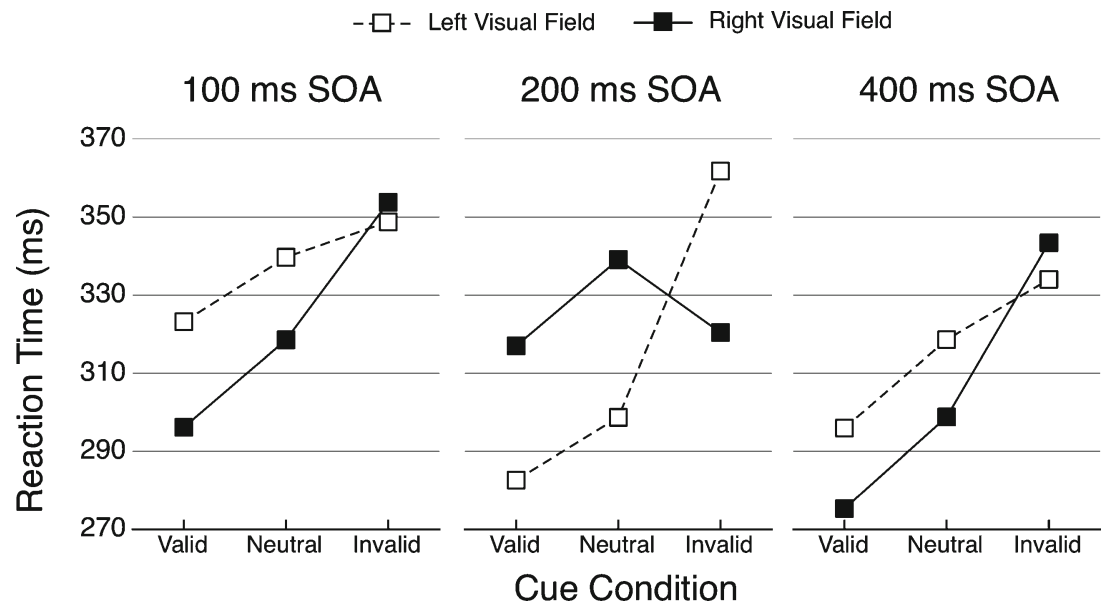

cue-target relationship, and visual field on $\operatorname{RTs}[F(4,80)<1$, $p=.72]$.

\section{Discussion}

Experiment 2 sought to replicate the results of Grubb and Reed (2002) in which participants were asked to respond to targets presented in the LVF or RVF using response keys that were spatially aligned with the LVF and RVF, respectively. Although the overall cuing effect was larger in Experiment 2 than in Experiment 1, the three-way interaction between trunk orientation, cue-target relationship, and visual field was not significant. As such, despite the similarities between Experiment 2 and Grubb and Reed (2002), we failed to replicate the finding for which these authors suggested trunk orientation elicited a disengagement effect in healthy control participants.
Fig. 7 Experiment 3 mean reaction times for each trunk orientation (panels: $30^{\circ}$ left, $15^{\circ}$ left, center, $15^{\circ}$ right, $30^{\circ}$ right), cue-target relationship (valid, neutral, invalid), and visual field (left, dotted line and open symbols; right, solid line and symbols)
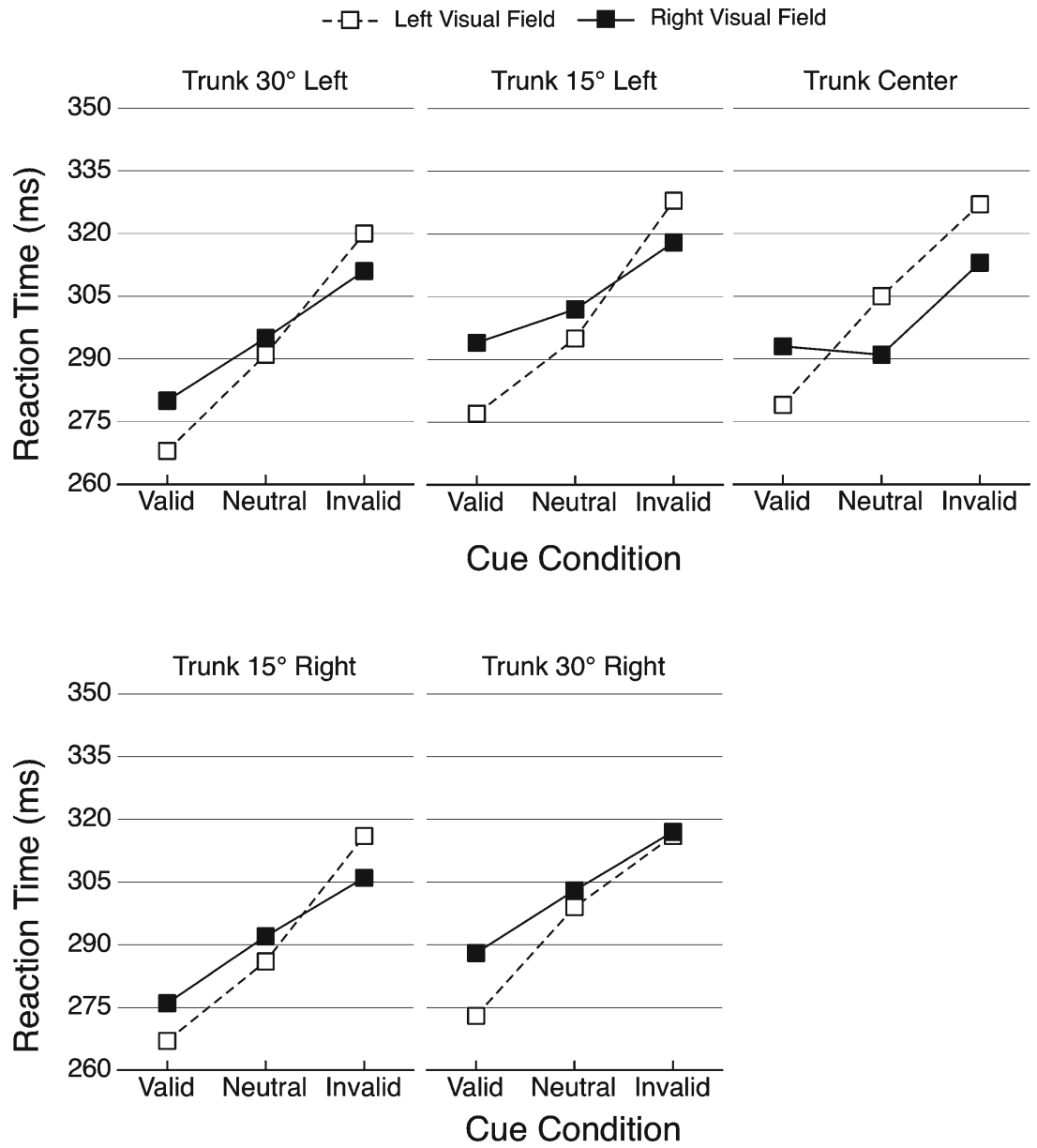
RTs for valid and neutral LVF targets were also slower than those for RVF targets, and RTs for invalidly cued targets in the RVF were faster than those for neutral targets in the same visual field. Because participants were asked to respond to the location of the target stimulus, with the location being the side on which the target appeared (left or right), it is possible that the LVF bias observed at this SOA was related to the right hemisphere's bias in processing visual information on a global level (for a review, see Hellige, 2006). We did not observe LVF biases for valid or neutral cues in Experiment 1 or 4 when participants were asked to respond to a more local feature of the target stimulus, color. Furthermore, in the subsequent Experiment 3, we found a similar trend of LVF bias for valid and neutral targets in four out of the five trunk rotation conditions (see Fig. 7 below), when participants were also asked to respond the spatial location of the target.

\section{Experiment 3}

Experiment 3 sought to once again replicate the pattern of results reported by Grubb and Reed (2002) by using spatially compatible motor responses (similar to Exp. 2). In contrast to Experiment 2, however, we employed a single 200ms SOA (the same SOA used by Grubb \& Reed, 2002). Additionally, we added a $30^{\circ}$ trunk rotation to each side, to rule out the possibility that our $15^{\circ}$ trunk rotation might have been too weak to elicit any effects on performance in our tasks.

\section{Method}

Participants A group of 20 naïve undergraduate students from the Department of Psychology at Dalhousie University (eight male, 13 female) participated in the experiment in exchange for partial course credit. The participants were right-handed and reported no history of visual, motor, or neurological abnormalities. All participants provided informed consent, and the project was approved by the Dalhousie University Research Ethics Board.

Procedure The procedure was the same that had been used in Experiment 2, except that a single 200-ms SOA was used and an additional $30^{\circ}$ trunk rotation was added to each side. Participants completed 500 trials in total (100 trials for each of the five trunk orientation blocks).

Data analysis Mean RTs were analyzed using a 2 (visual field: left, right) $\times 3$ (cue-target relationship: valid, neutral, invalid) $\times 5$ (trunk orientation: $30^{\circ}$ and $15^{\circ}$ left, center, $15^{\circ}$ and $30^{\circ}$ right) repeated measures ANOVA $(\alpha=.05)$. Significant main effects were analyzed post hoc using Bonferroni correction $(\alpha=.05)$. Greenhouse-Geisser-corrected $p$ values are reported.

\section{Results}

Figure 7 plots the average RTs for each trunk orientation (panels: $30^{\circ}$ left, $15^{\circ}$ left, center, $15^{\circ}$ right, $30^{\circ}$ right), cue-target relationship (valid, neutral, invalid), and visual field (LVF, dotted line and open symbols; RVF, solid line and symbols). Accuracy was high across the conditions $\left(M_{\mathrm{PC}}\right.$ : .964).

As expected, and consistent with Experiments 1 and 2, RTs varied with cue-target relationship, such that RTs were increasingly fast for valid $\left(M_{\mathrm{RT}}=279\right)$ versus neutral $\left(M_{\mathrm{RT}}=296\right)$ versus invalid $\left(M_{\mathrm{RT}}=317\right)$ cues $[F(2,38)=$ $\left.41.61, p=.001 ; M_{\text {Effect }}=38 \mathrm{~ms}\right]$. However, once again, the three-way interaction between trunk orientation, cue-target relationship, and visual field on RTs was not significant $[F(8,152)=1.49, p=.21]$.

\section{Discussion}

Unlike the results reported by Grubb and Reed (2002), but consistent with Experiments 1 and 2, the three-way interaction between trunk orientation, cue-target relationship, and visual field was not obtained in Experiment 3. However, as the response keys used in Experiment 3 were spatially aligned with visual field, this method once again confounded any effects of trunk orientation on covert attention with motor response preparation. For completeness, in Experiment 4 we sought to tease apart these two possible explanations by asking participants to respond to the color of the target (as in Exp. 1); the methodological similarities between Experiment 3 and Grubb and Reed's (2002) study were maintained in Experiment 4.

\section{Experiment 4}

In Experiment 4, participants reported the color of the target using response keys oriented orthogonally to the visual fields. All other aspects remained consistent with Experiment 3.

\section{Method}

Participants A group of 26 naïve undergraduate students from the Department of Psychology at Dalhousie University (nine male, 17 female) participated in the experiment in exchange for partial course credit. All of the participants 
were right-handed and reported no history of visual, motor, or neurological abnormalities. All participants provided informed consent, and the project was approved by the Dalhousie Research Ethics Board.

Procedure The task and procedure was identical to that used in Experiment 3, with the exception that participants responded to the color of the target using response keys oriented orthogonally to the visual fields (as in Exp. 1).

Results

Figure 8 plots average RTs for each trunk orientation (panels: left, center, right), cue-target relationship (valid, neutral, invalid), and visual field (LVF, dotted lines and open symbols; RVF, solid lines and symbols). Accuracy was high across conditions in Experiment 4 ( $M_{\mathrm{PC}}$. .960).

Consistent with our first three experiments, RTs were increasingly fast for valid $\left(M_{\mathrm{RT}}=404\right)$ versus neutral $\quad\left(M_{\mathrm{RT}}=416\right)$ versus invalid $\left(M_{\mathrm{RT}}=425\right)$ cues $[F(2,50)=39.71, p<.001$; $\left.M_{\text {Effect }}=22 \mathrm{~ms}\right]$. However, in Experiment 4 we once again failed to find a significant three-way interaction between trunk orientation, cue-target relationship, and visual field on RTs $[F(8,200)=1.30, p=.26]$.

Discussion

As we discussed earlier, the use of spatially aligned response keys in Experiment 3 was ambiguous with respect to the role of attentional or motor-based effects in the results. When responses to targets were not confounded with the locations of the targets in Experiment 4 (as in Exp. 1), we again found no significant interactions between trunk orientation, cuetarget relationship, and visual field. In Experiment 5, we strictly replicated the methodology of Grubb and Reed (2002).

\section{Experiment 5}

In Experiment 5, we directly replicated the methods of Grubb and Reed (2002); participants reported the location of targets using congruent, spatially aligned motor responses, once again confounding visual field and the direction of the motor
Fig. 8 Experiment 4 mean reaction times for each trunk orientation (panels: $30^{\circ}$ left, $15^{\circ}$ left, center, $15^{\circ}$ right, $30^{\circ}$ right), cue-target relationship (valid, neutral, invalid), and visual field (left, dotted line and open symbols; right, solid line and symbols)
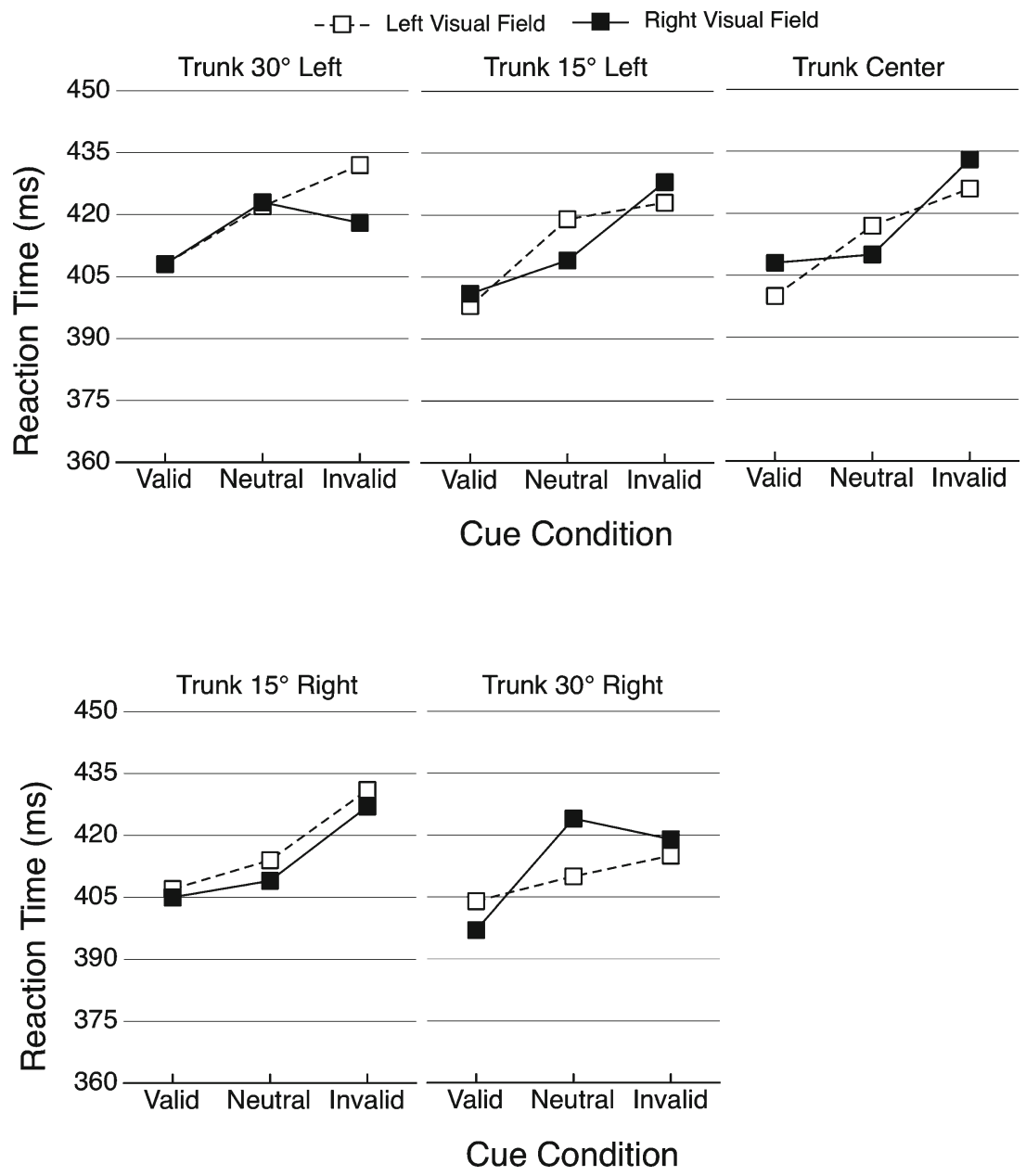
response. Three trunk orientations were included $\left(15^{\circ}\right.$ to the left or right and straight ahead), and a single 200-ms SOA. Furthermore, "neutral trials" consisted of a target preceded by no spatial cue, and valid and invalid spatial cues remained "bolded" until after the target had appeared and the response was provided. No spatial placeholders were used.

\section{Method}

Participants A group of 17 naïve undergraduate students from the Department of Psychology at Dalhousie University (one male, 16 females) participated in the experiment in exchange for partial course credit. Grubb and Reed had recruited 18 participants for their 2002 study. All of the participants were right-handed and reported no history of visual, motor or neurological abnormalities. All participants provided informed consent, and the project was approved by the Dalhousie Research Ethics Board.

Procedure The task and procedure was identical to that used in Grubb and Reed (2002). A brief description is presented below (see Grubb \& Reed, 2002). As in Experiment 1, participants performed a choice RT task with their trunk in one of three orientations (blocked trials, counterbalanced): $15^{\circ}$ leftward, straight ahead $\left(0^{\circ}\right)$, and $15^{\circ}$ rightward (relative to the computer monitor and head orientation). The response keys were mounted directly between the chinrest and the monitor. Participants completed 300 trials in total (100 trials for each trunk orientation block).

Choice reaction time task On each trial, participants responded to the location of a target (left or right) with their right hand by pressing one of two computer keyboard keys (the left or right arrow keys, respectively). Participants began by fixating a cross presented in the center of the computer monitor (measuring $2^{\circ}$ ). On the majority of trials, after a variable delay between 1,500 and $3,000 \mathrm{~ms}$, a cue box (black outline of a square, $2^{\circ}$ ) would appear $5^{\circ}$ to the left or right of the fixation cross. The target (an asterisk, $1.8^{\circ}$ ) would then appear $200 \mathrm{~ms}$ later. On $70 \%$ of the trials, the relationship between the cue and the target was valid. The remaining $30 \%$ of trials were divided into invalid ( $20 \%$ of trials) and no-cue trials (10\% of trials; as in Grubb \& Reed, 2002, these served as catch trials and were not analyzed). All stimuli were displayed using SR Research Experiment Builder 1.10.1 (SR Research Inc., Canada) on a multimedia computer system.

Data analysis The dependent measure of interest, RT (in ms), was analyzed using a 2 (visual field: left, right) $\times 2$ (cue-target relationship: valid, invalid) 3 (trunk orientation: $15^{\circ}$ left, center, $15^{\circ}$ right) repeated measures ANOVA $(\alpha=.05)$. Significant main effects were analyzed post hoc using Bonferroni correction. Greenhouse-Geisser-corrected $p$ values are reported.

\section{Results}

Figure 9 plots average RTs for each trunk orientation (panels: left, center, right), cue-target relationship (valid, invalid), and visual field (LVF, dotted lines and open symbols; RVF, solid lines and symbols). Accuracy was high across conditions in Experiment 5 ( $M_{\mathrm{PC}}$ : .98).

Consistent with our first four experiments, RTs were fastest for valid $\left(M_{\mathrm{RT}}=334\right)$, relative to invalid $\left(M_{\mathrm{RT}}=\right.$ $410)$, cues $\left[F(1,16)=183.86, p<.001, M_{\text {Effect }}=75 \mathrm{~ms}\right]$. A significant interaction between cue-target relationship and target location also revealed faster RTs for RVF targets $\left(M_{\mathrm{RT}}\right.$ $=402)$ than for LVF targets $\left(M_{\mathrm{RT}}=419\right)$ on invalidly cued trials only $[F(1,16)=10.36, p=.006]$. However, importantly, we did not find a significant three-way interaction between trunk orientation, cue-target relationship, and visual field on RTs $[F(2,32)<1, p=.60]$.

\section{Discussion}

Consistent with Experiments 1-4, we failed to find a threeway interaction between trunk orientation, target location, and cue-target relationship, despite our strict replication of
Fig. 9 Experiment 5 mean reaction times for each trunk orientation (panels: $15^{\circ}$ left, center, $15^{\circ}$ right), cue-target relationship (valid, invalid), and visual field (left, dotted line and open symbols; right, solid lines and symbols)

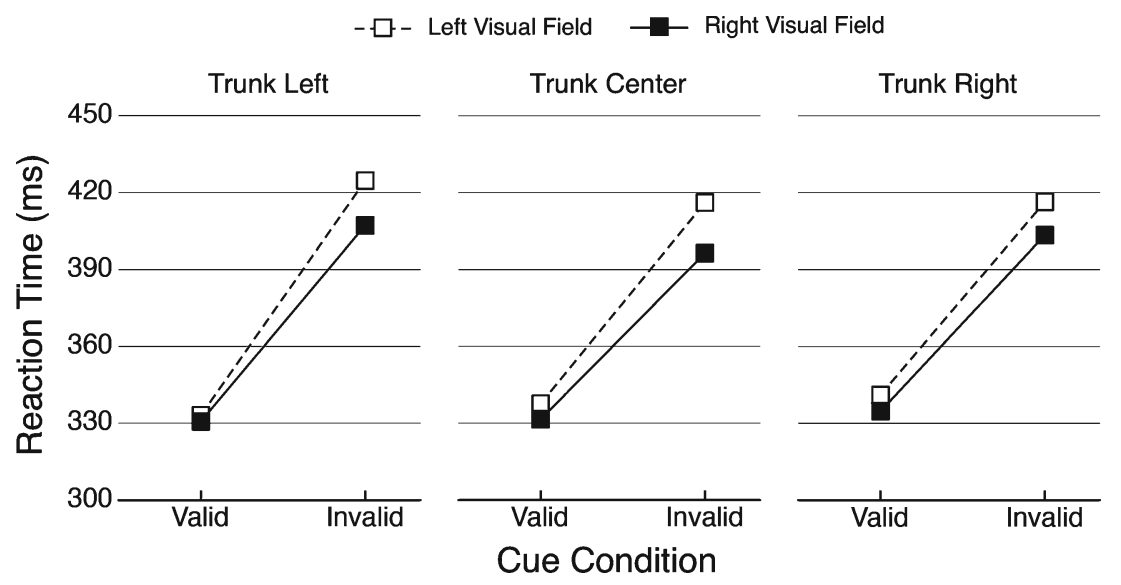


the methodology used by Grubb and Reed (2002). This result further substantiates the lack of support for a relationship between trunk rotation and the disengagement of spatial attention evidenced through the impaired detection of invalidly cued targets.

\section{General discussion}

Grubb and Reed (2002) reported an interaction between trunk orientation, cue-target relationship, and visual field such that changes in trunk orientation had no effect on the RTs for validly cued targets, but rotating the trunk to the left resulted in shorter RTs for invalidly cued targets in the LVF (ipsilateral to trunk orientation) than in the RVF (contralateral to trunk orientation). These authors concluded that trunk orientation biased the disengagement of attention and led to faster detection of stimuli presented on the side of space ipsilateral to the trunk orientation, similar to the disengagement effect inferred from excessively long RTs for invalidly cued targets in the contralesional half of space in patients suffering from neglect or extinction following damage to the right parietal lobe (Losier \& Klein, 2001; Posner et al., 1984).

However, because the precues not only signaled the likely location of targets, but also the likely correct response in Grubb and Reed (2002), a motor preparation mechanism could also have accounted for their results. Specifically, trunk orientation could have biased the generation of motor responses in the direction of the trunk rotation, leading to faster leftward responses in general. Here, we attempted to dissociate these two possible explanations in a series of four separate experiments during which participants responded to the color or location of targets using spatially congruent or orthogonal responses. Although we found that the pattern of results reported by Grubb and Reed (2002) could not be replicated, our results do not suggest that their original finding was due to a bias in the motor responses, but instead was spurious in nature.

In Experiments 1 and 4, participants' responses were not spatially aligned with the target locations. However, both experiments failed to replicate the significant three-way interaction between trunk orientation, cue-target relationship, and visual field reported by Grubb and Reed (2002). Nor could we replicate the findings of Grubb and Reed (2002) in Experiment 2 or 3, using their method, when the visual field and keypress responses were spatially congruent, even when we employed the same single 200-ms SOA in Experiment 3. Moreover, larger changes in trunk orientation $\left(30^{\circ}\right)$ and a reduction in the overall number of trials that participants were asked to complete failed to elicit this three-way interaction (although a reduction in the number of trials resulted in an effect of trunk orientation alone). In
Experiment 5, a strict replication of Grubb and Reed's methodology also failed to replicate their reported effect. Together, these results suggest that the specific effect of trunk orientation on performance as a function of cue-target relationship and visual field reported by Grubb and Reed (2002) may have been anomalous. It does not appear that changes in trunk orientation facilitate faster motor responses on the ipsilateral side of space or bias covert shifts in covert attention.

Our nonreplication of Grubb and Reed (2002) is not due to a failure to successfully capture participants' attention. Consistent with standard cuing effects, invalidly cued targets resulted in longer RTs than did validly cued targets in all of our experiments. Cue-target relationship effects have been previously reported in work demonstrating the benefit (i.e., decreased RTs, increased accuracy) associated with preceding stimuli with a valid warning signal (Posner, Klein, Summers, \& Buggie, 1973; Klein \& Kerr, 1974). Furthermore, Experiment 1 revealed no interactions between cue-target relationship and SOA, enabling us to infer that once participants' attention was captured, it correctly remained at the cued location, which was more likely than the opposite location to contain the target. In Experiment 2, however, we found that the effect of cue-target relationship varied with both SOA and visual field together. Although one might have expected the improvements in performance (i.e., faster RTs) across the invalid, neutral, and valid cue conditions to decrease with larger SOAs, we found that this was the case for left targets at the lowest and highest SOAs, but for right targets at the middle SOA.

In Experiments 1-4, we observed overall cuing effects that were smaller (range: 22 to $45 \mathrm{~ms}$ ) than those observed by Grubb and Reed (2002; approximately $70 \mathrm{~ms}$ ). Upon directly replicating the methodology of Grubb and Reed in Experiment 5 with 17 participants, we observed a much larger cuing effect $(75 \mathrm{~ms})$. However, the larger cuing effect was in the absence of a significant interaction between trunk orientation, cue-target relationship, and visual field, suggesting that our failure to replicate Grubb and Reed (2002) was not due to the size of the observed effects in Experiments 1-4.

Furthermore, using the effect size of the three-way interaction between trunk, cue-target relationship, and visual field found in Experiment $5\left(\eta_{\mathrm{p}}^{2}=.027\right)$, we determined that in the event that the three-way interaction effect reported by Grubb and Reed (2002) was not spurious, a sample size of 888 participants would have been required to obtain significance at the .05 level $(90 \%$ power, as computed using G*Power, version 3.1.3; Faul, Erdfelder, Lang, \& Buchner, 2007).

The five experiments included in this study have systematically examined the role of a body-centered reference frame on covert orienting in healthy participants. Overall, our results are consistent with those of Karnath et al. (1991, 1994), who suggested that the trunk and vestibular effects 
observed in participants with HSN likely targeted a deficit in their body-centered representation of space (i.e., the egocentric representation of body position with respect to objects in the environment), as opposed to general effects on the covert orienting of spatial attention (e.g., Bisiach et al. 1985; Karnath, 1994; Karnath et al., 1991; Rorden et al. 2001). These authors found that leftward trunk rotations, caloric stimulation to the left ear, and left neck tendon vibration ameliorated a rightward bias in estimates of perceptual straight-ahead in patients with HSN (Karnath, 1994; Karnath et al. 1991).

\section{Summary and conclusions}

The results of all five of our experiments strongly suggest that the three-way interaction between trunk orientation, cue-target relationship, and visual field reported by Grubb and Reed (2002) was spurious. In fact, on the basis of our findings, any interaction effects between cue-target relationship and visual field do not appear to be affected by trunk orientation, and as such would have little to do with the disengagement of attention. Furthermore, the inability to replicate Grubb and Reed (2002) was not due to an inability to successfully capture or maintain participants' attention using pretarget cues, to the magnitude of the trunk orientation changes, to the use of more than one SOA, or to the overall amount of time that participants spent completing our tasks. Our results support the suggestion that the trunk rotation effects observed in participants with HSN target a deficit in their body-centered representation of space (e.g., Karnath, 1994; Karnath et al., 1991).

\section{References}

Andersen, R. A., Snyder, L. H., Bradley, D. C., \& Xing, J. (1997). Multimodal representation of space in the posterior parietal cortex and its use in planning movements. Annual Review of Neuroscience, 20, 303-330. doi:10.1146/annurev.neuro.20.1.303

Avillac, M., Denève, S., Olivier, E., Pouget, A., \& Duhamel, J.-R. (2005). Reference frames for representing visual and tactile locations in parietal cortex. Nature Neuroscience, 8, 941-949.

Bartolomeo, P., \& Chokron, S. (1999). Egocentric frame of reference: Its role in spatial bias after right hemisphere lesions. Neuropsychologia, 37, 881-894. doi:10.1016/S0028-3932(98)00150-X

Bartolomeo, P., \& Chokron, S. (2002). Orienting of attention in left unilateral neglect. Neuroscience \& Biobehavioral Reviews, 26, 217-234.

Batista, A. P., Santhanam, G., Yu, B. M., Ryu, S. I., Afshar, A., \& Shenoy, K. V. (2007). Reference frames for reach planning in macaque dorsal premotor cortex. Journal of Neurophysiology, 98, 966-983. doi:10.1152/jn.00421.2006

Bisiach, E., Capitani, E., \& Porta, E. (1985). Two basic properties of space representation in the brain: evidence from unilateral neglect. Journal of Neurology, Neurosurgery \& Psychiatry, 48, 141-144.

Bowen, A., McKenna, K., \& Tallis, R. C. (1999). Reasons for variability in the reported rate of occurrence of unilateral spatial neglect after stroke. Stroke, 30, 1196-1202.
Buneo, C. A., Jarvis, M. R., Batista, A. P., \& Andersen, R. A. (2002). Direct visuomotor transformations for reaching. Nature, 416, 632-636.

Butler, B. C., Lawrence, M., Eskes, G. A., \& Klein, R. (2009). Visual search patterns in neglect: Comparison of peripersonal and extrapersonal space. Neuropsychologia, 47, 869-878. doi:10.1016/ j.neuropsychologia.2008.12.020

Caminiti, R., Johnson, P. B., Galli, C., Ferraina, S., \& Burnod, Y. (1991). Making arm movements within different parts of space: the premotor and motor cortical representation of a coordinate system for reaching to visual targets. Journal of Neuroscience, 11, 1182-1197.

Culham, J. C., \& Kanwisher, N. G. (2001). Neuroimaging of cognitive functions in human parietal cortex. Current Opinion in Neurobiology, 11, 157-163.

De Renzi, E. (1982). Disorders of space exploration and cognition. Chichester, UK: Wiley.

Faul, F., Erdfelder, E., Lang, A.-G., \& Buchner, A. (2007). G*Power 3: A flexible statistical power analysis program for the social, behavioral, and biomedical sciences. Behavior Research Methods, 39, 175-191. doi:10.3758/BF03193146

Graziano, M. S. A., \& Gross, C. G. (1998). Spatial maps for the control of movement. Current Opinion in Neurobiology, 8, 195-201.

Grubb, J. D., \& Reed, C. L. (2002). Trunk orientation induces neglectlike lateral biases in covert attention. Psychological Science, 13, $553-556$.

Hellige, J. B. (2006). Hemispheric asymmetry for visual information processing. Acta Neurobiologiae Experimentalis, 56, 485-497.

Jones, S. A. H., \& Henriques, D. Y. P. (2010). Memory for proprioceptive and multisensory targets is partially coded relative to gaze. Neuropsychologia, 48, 3782-3792. doi:10.1016/j.neuropsychologia. 2010.10.001

Karnath, H.-O. (1994). Subjective body orientation in neglect and the interactive contribution of neck muscle proprioception and vestibular stimulation. Brain, 117, 1001-1012.

Karnath, H.-O., Schenkel, P., \& Fischer, B. (1991). Trunk orientation as the determining factor of the "contralateral" deficit in the neglect syndrome and as the physical anchor of the internal representation of body orientation in space. Brain, 114, 19972014.

Klein, R. M. (2004). On the control of visual orienting. In M. I. Posner (Ed.), Cognitive neuroscience of attention (pp. 29-44). New York, NY: Guilford Press.

Klein, R. M., \& Kerr, B. (1974). Visual signal detection and the locus of foreperiod effects. Memory \& Cognition, 2, 431-435.

Lacquaniti, F., Guigon, E., Bianchi, L., Ferraina, S., \& Caminiti, R. (1995). Representing spatial information for limb movement: Role of area 5 in the monkey. Cerebral Cortex, 5, 391-409.

Losier, B. J. W., \& Klein, R. M. (2001). A review of evidence for a disengage deficit following parietal lobe damage. Neuroscience \& Biobehavioral Reviews, 25, 1-13.

Mountcastle, V. B., Lynch, J. C., Georgopoulos, A., Sakata, H., \& Acuna, C. (1975). Posterior parietal association cortex of the monkey: Command functions for operations within extrapersonal space. Journal of Neurophysiology, 38, 871-908.

Posner, M. I., Klein, R., Summers, J., \& Buggie, S. (1973). On the selection of signals. Memory \& Cognition, 1, 2-12. doi:10.3758/ BF03198062

Posner, M. I., Walker, J. A., Friederiech, F. J., \& Rafal, R. D. (1984). Effects of parietal injury on covert orienting of attention. Journal of Neuroscience, 4, 1863-1974.

Previc, F. H. (1990). Functional specialization in the lower and upper visual fields in humans: Its ecological origins and neurophysiological implications. Behavioral and Brain Sciences, 13, 519-575.

Previc, F. H. (1998). The neuropsychology of 3-D space. Psychological Bulletin, 124, 123-164. doi:10.1037/0033-2909.124.2.123 
Rorden, C., Karnath, H.-O., \& Driver, J. (2001). Do neck-proprioceptive and caloric-vestibular stimulation influence covert visual attention in normals, as they influence visual neglect? Neuropsychologia, 39, 364-375. doi:10.1016/S0028-3932(00) 00126-3

Spence, C., \& Driver, J. (1997). Audiovisual links in endogenous covert spatial orienting. Perception \& Psychophysics, 59, 122. doi:10.3758/BF03206843
Wandell, B. A. (1999). Computational neuroimaging of human visual cortex. Annual Review of Neuroscience, 22, 145-173.

Wu, W., \& Hatsopoulos, N. (2006). Evidence against a single coordinate system representation in the motor cortex. Experimental Brain Research, 175, 197-210.

Wu, W., \& Hatsopoulos, N. G. (2007). Coordinate system representations of movement direction in the premotor cortex. Experimental Brain Research, 176, 652-657. 